\title{
Does modification of portal venous blood flow using balloon-occluded retrograde transvenous obliteration affect the mucosa of the small intestine?
}

Takayuki Yokota $^{1}$, Shinichi Hashimoto ${ }^{1 *}$, Ryo Kawasato ${ }^{1}$, Tomohiro Shirasawa ${ }^{1}$, Satoko Kaneyama ${ }^{1}$, Atsushi Goto ${ }^{1}$, Takuya Iwamoto ${ }^{1}$, Tsuyoshi $^{1}$ Ishikawa $^{1}$, Takeshi Okamoto ${ }^{1}$, Jun Nishikawa ${ }^{2}$ and Isao Sakaida ${ }^{1}$

${ }^{1}$ Department of Gastroenterology and Hepatology, Yamaguchi University Graduate School of Medicine, Yamaguchi, Japan

${ }^{2}$ Department of Laboratory Science, Yamaguchi University Graduate School of Medicine, Yamaguchi, Japan

\begin{abstract}
Objective: Portal hypertensive enteropathy may be evaluated using capsule endoscopy (CE), but the pathological findings are still unclear. After balloon-occluded retrograde transvenous obliteration (B-RTO), which is usually performed for the treatment of gastric varices and hepatic encephalopathy in patients with liver cirrhosis, occlusion of portosystemic shunts causes an increase in portal blood pressure. This study assessed the effects of this increase on the mucosa of the small intestine.

Material and methods: We carried out a retrospective comparison of CE findings in 9 patients with liver cirrhosis who underwent B-RTO. CE was performed preand post-procedure with an interval of 29-52 days and changes in red spot, angioectasia, varices, and inflammatory-like lesions were evaluated.

Results: The red spot score increased in 6 patients after B-RTO and was unchanged in 3. The angioectasia score increased in 4 patients and was unchanged in 5. No varices were observed either before or after B-RTO. The score for inflammatory-like lesions increased in 4 patients, remained unchanged in 1 patient, and decreased in 4 patients. The changes in the scores of red spot and angioectasia after B-RTO were statistically significant $(\mathrm{p}<0.05)$.

Conclusions: The increase in portal blood pressure caused by B-RTO results in changes in the mucosa of the small intestine.
\end{abstract}

\section{Introduction}

Among patients with portal hypertension, such as those with liver cirrhosis, 63-98\% will develop portal hypertensive gastropathy and 48.6-93\% portal hypertensive colopathy, as well as esophagogastric or rectal varices $[1,2]$. On the other hand, little information has been reported regarding portal hypertensive enteropathy (PHE) with mucosal alteration in the small bowel. The introduction of capsule endoscopy (CE) has enabled us to obtain information about the mucosa of the small intestine in a less invasive manner, and some reports of findings from the mucosa of the small intestine in patients with portal hypertension or liver cirrhosis have been published recently [3-10].

Transjugular intrahepatic portosystemic shunt (TIPS) is a procedure used to lower portal blood pressure. Matsushita et al. reported that portal blood pressure was an important factor for changes in the mucosa of the small intestine, as CE performed before and after TIPS showed improved red spot, edema, and varices in the small bowel post procedure [11].

While balloon-occluded retrograde transvenous obliteration (B-RTO) is a useful procedure for treating gastric varices or hepatic encephalopathy, the increase in portal blood pressure after B-RTO is of concern because of the occlusion of portosystemic shunts [12], while aggravation of esophageal varices or ascites/pleural effusion has also been reported [13-15]. However, no study to date has examined the effect of changes in portal blood pressure caused by B-RTO on the mucosa of the small intestine. Therefore, the aim of our study was to retrospectively evaluate changes in the mucosa of the small intestine, identified by $\mathrm{CE}$, caused by the alterations in portal vein hemodynamics following B-RTO.

\section{Materials and methods}

\section{Patients}

Between September 2010 and September 2014, 42 patients underwent B-RTO at our hospital. CE was performed before and after B-RTO in 9 of these 42 patients. This retrospective study analyzed the CE information from these 9 patients. The study was approved by the ethical committee on human research of Yamaguchi University Hospital.

\section{CE procedures}

CE was performed within 5 days before the B-RTO procedure and was repeated after the procedure. The technical details of the CE

Correspondence to: Dr. Shinichi Hashimoto, Department of Gastroenterology and Hepatology, Yamaguchi University Graduate School of Medicine, 1-1-1 Minami-Kogushi, Ube, Yamaguchi 755-8505, Japan, Tel: +81-836-22-2241, Fax: +81-836-22-2240, E-mail: has-333@yamaguchi-u.ac.jp

Key words: balloon-occluded retrograde transvenous obliteration, capsule endoscopy, portal hypertensive enteropathy

Received: April 26, 2016; Accepted: May 27, 2016; Published: May 31, 2016 
procedure and the associated risks were explained and all patients provided written informed consent. The PillCam SB CE system was used (Given Imaging Ltd., Yokneam, Israel) and the image analysis was performed using the Rapid Reader software (version 5) from the same company. The procedures for CE and analysis have been reported previously [16].

Gastric transit time (GTT) was defined as the time between capsule arrival in the stomach and arrival in the duodenum. Small bowel transit time (SBTT) was defined as the time between capsule arrival in the duodenum and arrival in the cecum. For evaluation, the small intestine was divided into 3 regions, defined by dividing the SBTT into 3 segments, designated as regions 1, 2 and 3 in descending order from the oral side of the small intestine.

\section{B-RTO procedure and measurement of wedged hepatic venous pressure}

The B-RTO procedure was performed according to the original method described fully by Kanagawa et al. [17]; we simply followed the procedural protocol, as previously described [18]. During these procedures, 4000 units of human haptoglobin were given intravenously to prevent renal failure related to hemolysis.

In this study, the wedged hepatic venous pressure (WHVP) was used as a surrogate measure of portal blood pressure [19]. WHVP was measured using the balloon catheter, before and after B-RTO, as we have previously reported [18].

\section{Classification of CE findings for the small intestine}

PHE-related CE findings were classified into red spots, angioectasia and varices, based on the classification system of De Palma et al. [9] and Abdelaal et al. [6]. According to this classification system, mucosal edema, erythema and granularity were defined as inflammatory-like lesions. Examples of the different classification of findings are shown in Figure 1. The scoring system reported by Matsushita et al. [11] was
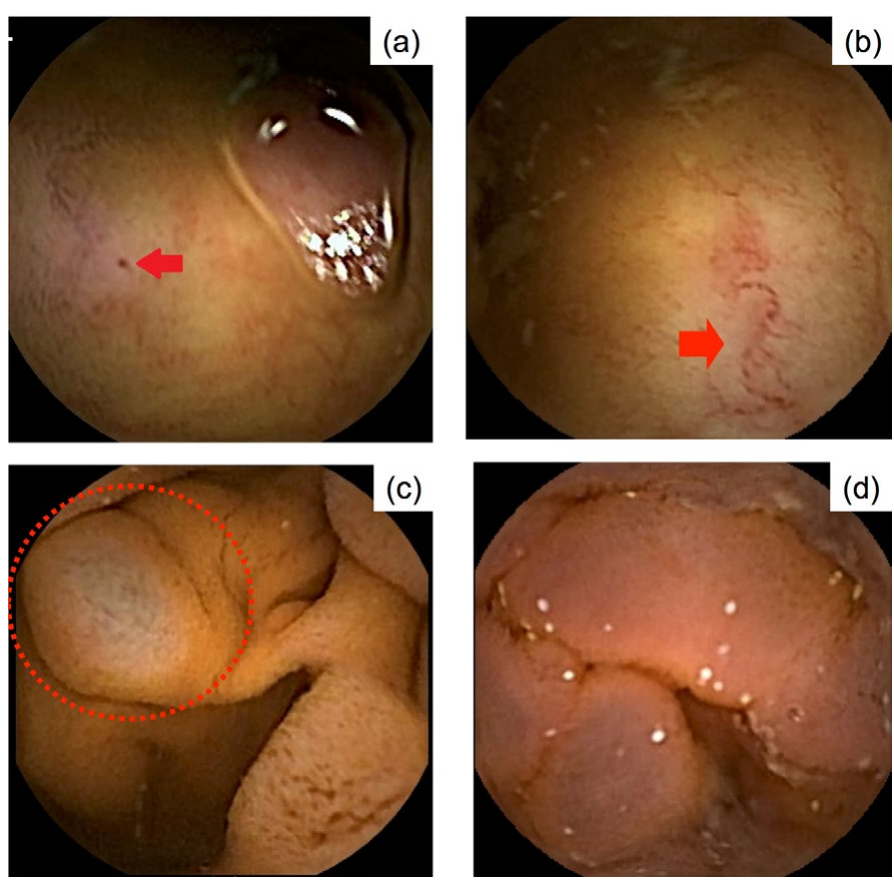

Figure 1. Capsule endoscopy of the small intestine. a red spot, b angioectasia, c SB varices, and $\mathbf{d}$ inflammatory-like lesion. modified to report the number (i.e., count) of red spots, angioectasias and varices in the entire small intestine, as follows: ' 0 ', no identifiable lesion; ' 1 ', identification of 1-5 lesions; ' 2 ', identification of 6-10 lesions; ' 3 ', identification of 11-15 lesions; and ' 4 ', identification of $>16$ lesions. The presence of inflammatory-like lesions was scored as either ' 0 ', indicative of negative findings, or ' 1 ', indicative of positive findings. The scores within each region of the small intestine were summed to provide a total score for inflammatory-like lesions.

\section{Statistical analysis}

Quantitative data were expressed as median (range) or mean \pm standard deviation. Changes in GTT and SBTT from before to after B-RTO were evaluated using paired $t$-tests, while changes in the scores for red spots, angioectasia, varices, and inflammatory-like lesions were evaluated using the Wilcoxon signed-rank test. A $p$-value $<0.05$ was considered to be statistically significant. All statistical analyses were performed using STATA13 (StataCorp LP, Texas, USA).

\section{Results}

\section{Patient characteristics}

Our study group included 5 male and 4 female patients, with a median age of 71 years (range, 53-86 years). Relevant characteristics of our study group are summarized in Table 1. All patients had liver cirrhosis due to various underlying causes: hepatitis $C$ virus $(n=4)$, alcohol $(n=2)$, hepatitis B virus $(n=1)$, non-alcoholic steatohepatitis $(\mathrm{n}=1)$, and hepatitis $\mathrm{C}$ virus in combination with alcohol $(\mathrm{n}=1)$. The indications for B-RTO were gastric varices in 5 patients and hepatic encephalopathy in 4 patients. The interval between the first and second CE ranged from 29 to 52 days, with a median of 37 days. Oral medications used included antihypertensive drugs $(n=5)$, diuretics $(\mathrm{n}=3)$, anticoagulants $(\mathrm{n}=3)$, and non-steroidal anti-inflammatory drugs $(\mathrm{n}=1)$.

\section{B-RTO}

Shunt occlusion was performed successfully in all patients, with no serious complications reported. The Child-Pugh score improved after B-RTO in 6 patients, remained unchanged in 2 patients and worsened in 1 patient. WHVP was measured at the time of B-RTO and after B-RTO in 4 of the 9 patients, with an increase in WHVP identified in all 4 patients (Figure 2).

\section{CE procedure}

CE successfully reached the cecum, within the battery-effective

Table 1. Patient characteristics, including indications for B-RTO and interval between preand post-capsule endoscopy procedures.

\begin{tabular}{|l|c|}
\hline Age (years) & 71 (range 53-86) \\
\hline Sex (male/female) & $5 / 4$ \\
\hline Etiology of LC & 4 \\
\hline Hepatitis C virus & 2 \\
\hline Alcohol & 1 \\
\hline Hepatitis B virus & 1 \\
\hline Hepatitis C virus + alcohol & 1 \\
\hline Non-alcoholic steatohepatitis & 5 \\
\hline Indication & 4 \\
\hline Gastric varices & 37 (range 29-52) \\
\hline Hepatic encephalopathy & \\
\hline Capsule endoscopy interval (days) & \\
\hline
\end{tabular}

B-RTO, balloon-occluded retrograde transvenous obliteration; LC, liver cirrhosis. 
hours, in all patients for both before and after B-RTO assessments, with observation of the entire small intestine available for analysis. There were no adverse events, such as aspiration or retention, associated with the CE procedure. The GTT was comparable before and after B-RTO, with mean GTT values of $45.1 \mathrm{~min}$ before B-RTO and $24.4 \mathrm{~min}$ after B-RTO. SBTT was significantly prolonged after B-RTO, with a mean SBTT of $306.7 \mathrm{~min}$ before compared to $409.0 \mathrm{~min}$ after B-RTO (Table 2).

\section{CE findings}

CE findings are reported in Table 3. The following before-to-after B-RTO changes in scores were identified: increased score for red spots in 6 patients, with the score remaining unchanged in the other 3 patients; increased score for angioectasia in 4 patients, the score remaining unchanged in 5 patients; and increased score for inflammatory-like lesions in 4 patients, the score remaining unchanged in 1 patient and decreasing in 4 patients. No varices were identified either before or after the B-RTO procedure. The statistical evaluation of change scores for CE findings, before and after B-RTO, is reported in Table 4, with significant change in score identified for red spots and angioectasia.

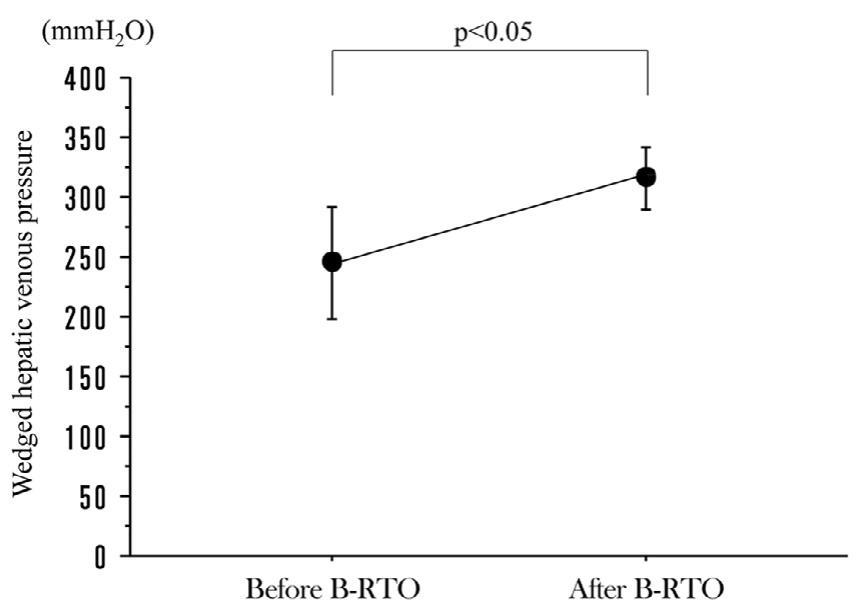

Figure 2. Wedged hepatic venous pressure (WHVP), before and after balloonoccluded transfemoral obliteration (B-RTO). WHVP was significantly increased after B-RTO. Data represent mean values \pm SE.

Table 2. Comparison of GTT and SBTT before and after B-RTO.

\begin{tabular}{|l|l|l|l|}
\hline & Before B-RTO & After B-RTO & $\boldsymbol{p}$-value \\
\hline GTT & $45.1 \pm 54.5$ & $24.4 \pm 20.0$ & 0.3738 \\
\hline SBTT & $306.7 \pm 161.0$ & $409.0 \pm 137.9$ & $0.0339^{*}$ \\
\hline
\end{tabular}

" $p<0.05$; B-RTO, balloon-occluded retrograde transvenous obliteration; GTT, gastric transit time; SBTT, small bowel transit time.

Table 3. Changes in scores of CE findings before and after B-RTO.

\begin{tabular}{|c|c|c|c|c|c|c|c|c|}
\hline \multirow[b]{2}{*}{ Patient } & \multicolumn{2}{|c|}{ Red spot } & \multicolumn{2}{|c|}{ Angioectasia } & \multicolumn{2}{|c|}{ Varices } & \multicolumn{2}{|c|}{ Inflammatory-like lesions } \\
\hline & Before B-RTO & After B-RTO & Before B-RTO & After B-RTO & Before B-RTO & After B-RTO & Before B-RTO & After B-RTO \\
\hline 1 & 1 & 1 & 1 & 1 & 0 & 0 & 1 & 0 \\
\hline 2 & 1 & 2 & 0 & 1 & 0 & 0 & 1 & 1 \\
\hline 3 & 0 & 1 & 1 & 1 & 0 & 0 & 1 & 2 \\
\hline 4 & 1 & 1 & 1 & 1 & 0 & 0 & 2 & 1 \\
\hline 5 & 1 & 2 & 1 & 1 & 0 & 0 & 2 & 3 \\
\hline 6 & 3 & 3 & 2 & 2 & 0 & 0 & 3 & 2 \\
\hline 7 & 0 & 2 & 1 & 3 & 0 & 0 & 2 & 3 \\
\hline 8 & 1 & 2 & 2 & 3 & 0 & 0 & 1 & 3 \\
\hline 9 & 2 & 3 & 2 & 4 & 0 & 0 & 3 & 2 \\
\hline
\end{tabular}

CE, capsule endoscopy; B-RTO, balloon-occluded retrograde transvenous obliteration.

\section{Long-term CE findings after B-RTO}

Long-term follow-up data were available for 1 patient (patient 1 in Table 3) who underwent assessment of the small intestine for anemia 3 years after B-RTO. CE identified the development of small intestinal varices, which had not been observed previously. Contrast-enhanced computed tomography also indicated the formation of varicose veins at the same site (Figure 3).

\section{Discussion}

The aim of our study was to evaluate the effects of altered portal vein hemodynamics, caused by B-RTO, on the mucosa of the small intestine. A significant increase in red spots and angioectasia was identified, indicating that altered portal blood pressure is an important risk factor for the formation of mucosal lesions of the small intestine. The increase in WHVP in all 4 patients in whom pressure was measured is consistent with findings of an increase in portal blood pressure after B-RTO measured with endoscopic ultrasound [20]. Our results also agree with those of previous reports that described an association between liver cirrhosis, which is a known cause of increased portal vein pressure, and the development of mucosal lesions in the small intestine [3-8]. De Palma et al. classified findings from the CE assessment of edema, erythema, granularity, and friability as mucosal inflammatory-like lesions, with vascular lesions defined as cherry red spots, telangiectasia- and angiodysplasia-like lesions, and varices [9]. Abdelaal et al. further classified erythema and edematous villi as mucosal inflammatory changes, defining vascular lesions as red spots, angioectasia, and small intestine varices [6]. Using a scoring system modified from Matsushita et al. [11], we reported a significant increase in the score for red spots and angioectasia after B-RTO, which we suggest is indicative of the involvement of increased portal blood pressure in the aggravation of vascular lesions of the mucosa of the small intestine. However, Matsushita et al. failed to identify a significant decrease in angiodysplasia-like lesions, despite lowering of portal blood pressure using a TIPS procedure [11]. The difference in the follow-up interval for CE assessment of 2 weeks in the study of Matsushita et al. and the 1 to 2 months in our study may explain these differences, as it may take some time for the effect of portal blood pressure to be manifested in the vascular status of the small intestine.

In the relatively short study period of 1 to 2 months, we did not find any evidence of the development of varices in our cohort. However, an incidental long-term follow-up of 1 patient in our study group provided evidence that increased portal blood pressure may be an important factor for the formation of small-intestinal varices as a long-term consequence of B-RTO (Figure 3). Although this evidence is weak, we do recommend a careful longer-term follow-up for patients after B-RTO to identify complications of small intestinal varices. 
Table 4. Comparison of CE findings in the small intestine before and after B-RTO.

\begin{tabular}{|l|c|c|c|}
\hline & Before B-RTO & After B-RTO & $\boldsymbol{p}$-value \\
\hline Red spot & $1.1 \pm 0.9$ & $1.9 \pm 0.8$ & $0.0158^{*}$ \\
\hline Angioectasia & $1.2 \pm 0.7$ & $1.9 \pm 1.2$ & $0.0474^{*}$ \\
\hline Varices & 0 & 0 & \\
\hline Inflammatory-like lesions & $1.8 \pm 0.8$ & $1.9 \pm 1.1$ & 0.8026 \\
\hline
\end{tabular}

" $p<0.05$; CE, capsule endoscopy; B-RTO, balloon-occluded retrograde transvenous obliteration.
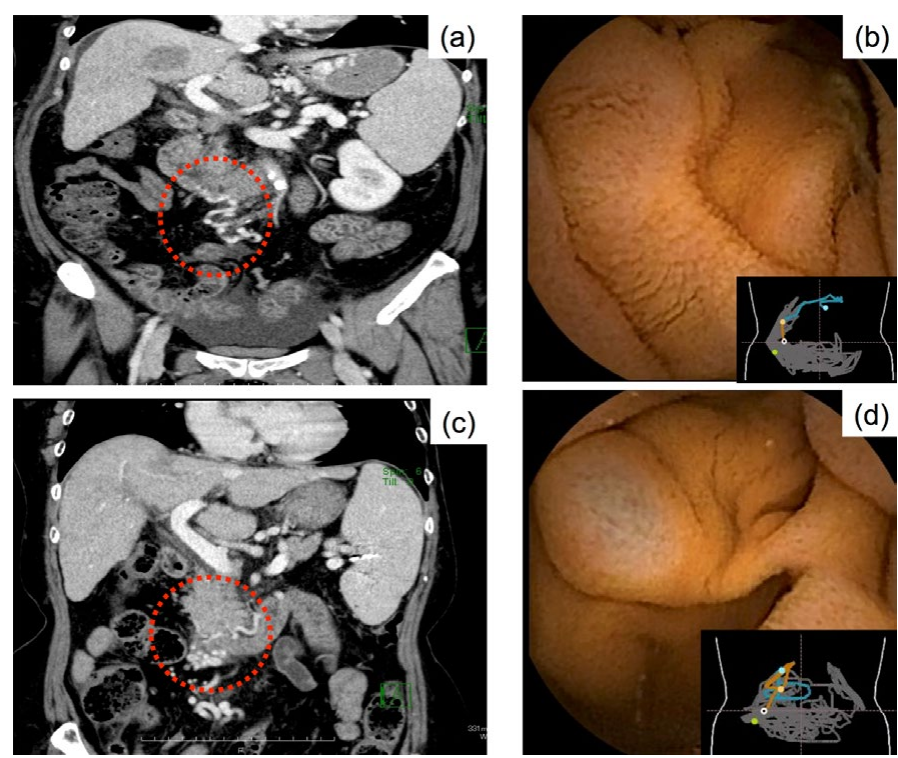

Figure 3. Capsule endoscopy performed as a component of the clinical evaluation for anemia in a 60-year-old woman (patient number 1, Table 3), 3 years after B-RTO. The image shows formation of varicose veins, which were not apparent on assessment 52 days after the surgical intervention: a slight vasodilation in the inferior duodenal flexure (red circle with dashed line) identified on a contrast-enhanced computed tomography (CT) image 52 days after B-RTO; b capsule endoscopy, performed 52 days after B-RTO, did not identify a varicose vein at the same site; $\mathbf{c}$ apparent venous dilatation observed (red circle with dashed line) on the CT image obtained 3 years after B-RTO.

In our study, we did not identify a significant effect of B-RTO on inflammatory-like lesions of the small intestine. Matsushita et al. [11] reported that TIPS significantly improved edema in the small bowel, while another report indicated that portal blood pressure was involved in intestinal edema [21]. Difficulty in reliably scoring the severity of mucosal edema when the small intestine is divided into 3 segments for analysis may explain our finding of no aggravation of inflammatorylike lesions in our study. Thus, development of a classification system for mucosal edema of the small intestine may be required to standardize the CE assessment. In addition, we need to consider that B-RTO improves hepatic function by increasing portal blood flow [22, 23], with 6 of 9 patients (66.6\%) in our study showing improvement in the Child-Pugh score after B-RTO. Therefore, as with portal blood pressure, changes in hepatic function may also be involved in the formation of inflammatory-like lesions. Further research is required to clarify this relationship.

The small intestine was divided into 3 regions to evaluate the distribution of red spots, angioectasia and inflammatory-like lesions. More lesions were observed in the 'oral' segment of the small intestine than in the other two regions; however, this between-region difference was not significant, possibly because of the small number of patients in our study group (data not shown). In the case of the patient who had a follow-up assessment 3 years after B-RTO, varices were also identified in the duodenum. A previous report has similarly indicated a higher number of lesions in the oral side of the small intestine in patients with portal hypertension [8], indicative of a possible selective sensitivity of the oral side of the small intestine to the effects of increased portal blood pressure. Based on the above evidence, observation of the deep duodenum at the time of esophagogastroduodenoscopy, performed after B-RTO, may be useful for the early detection of complications related to B-RTO.

Balloon endoscopy (BE) is a widely used method to evaluate the mucosa of the small intestine. In a study using BE, lesions of the mucosa of the small intestine were identified in $38.1 \%$ of patients with liver cirrhosis, with 'some type' of small-intestinal lesion identified in all patients with portal hypertension [24]. In another study, erythema, which was defined as a red spot image without bulge or defect of mucosa, telangiectasia and mucosal edema were identified in $24 \%, 5 \%$ and $38 \%$, respectively, of patients with portal hypertension [25]. Among the lesions identified, villous shortening with edema and edema showing telangiectasia were reported as having a 'herring roe appearance' and were regarded as characteristic findings of PHE [23]. In contrast to $\mathrm{CE}, \mathrm{BE}$ has the advantage of allowing tissue biopsy for histopathology. However, routine use of BE for PHE screening is impractical as the procedure is highly invasive and requires sedation [26]. Although CE carries the risk of retention, additional indications for $\mathrm{CE}$ have recently been approved in Japan and the safety of CE has also been increased by the introduction of the patency capsule. Therefore, CE should be actively recommended for small bowel screening in patients with liver cirrhosis.

The limitations of this study are as follows. First and foremost, this was a single-site, retrospective study with a small clinical population. A multicenter collaborative study, enrolling a larger population, is required to obtain evidence of the effects of B-RTO on the mucosa of the small intestine that is sufficiently clear to inform practice. In addition, CE should be performed at multiple time points to elucidate the appropriate follow-up duration after B-RTO; the interval for CE assessment in our study varied from 29 to 52 days. Despite these limitations, our study is the first to report on the changes in the mucosa of the small intestine caused by increased portal blood pressure after B-RTO using CE.

\section{Conclusions}

The main finding of this study is that an increase in portal blood pressure after B-RTO may lead to changes in the mucosa of the small intestine due to aggravation of vascular lesions, including red spots and angioectasia. While limited by the small size of the study, this evidence relating small intestine lesions to portal blood pressure has clear implications for practice, including the recommendation for a longterm follow-up of patients who have undergone a B-RTO procedure, as well as those with liver cirrhosis, using CE. A larger, multicenter study will be required to elucidate the pathological pathway relating portal hypertension to small intestine disease.

\section{Authorship and contributorship}

Yokota $\mathrm{T}$ drafted the manuscript. Hashimoto $\mathrm{S}$ performed data analysis and interpretation. Kawasato R, Shirasawa T, Kaneyama S, Goto A, Iwamoto T, Ishikawa T, Okamoto T and Nishikawa J contributed to the conception and design of the study. Sakaida I made final approval of the manuscript. 


\section{Acknowledgments}

We would like to thank Editage (www.editage.jp) for English language editing.

\section{Funding information}

None.

\section{Conflict of interest}

The authors declare that there is no conflict of interest regarding the publication of this paper.

\section{Trial registration no.: UMIN 000019579.}

\section{References}

1. Piqué JM (1997) Portal hypertensive gastropathy. Clin Gastroenterol 11: 257-270.

2. Burak KW, Lee SS, Beck PL (2001) Portal hypertensive gastropathy and gastric antral vascular ectasia (GAVE) syndrome. Gut 49: 866-872. [Crossref]

3. Goulas S, Triantafyllidou K, Karagiannis S, Nicolaou P, Galanis P, et al. (2008) Capsule endoscopy in the investigation of patients with portal hypertension and anemia. Can J Gastroenterol 22: 469-474. [Crossref]

4. Canlas KR, Dobozi BM, Lin S, Smith AD, Rockey DC, et al. (2008) Using capsule endoscopy to identify GI tract lesions in cirrhotic patients with portal hypertension and chronic anemia. J Clin Gastroenterol 42: 844-848. [Crossref]

5. Figueiredo P, Almeida N, Lérias C, Lopes S, Gouveia H, et al. (2008) Effect of portal hypertension in the small bowel: an endoscopic approach. Dig Dis Sci 53: 2144-2150. [Crossref]

6. Abdelaal UM, Morita E, Nouda S, Kuramoto T, Miyaji K, et al. (2010) Evaluation of portal hypertensive enteropathy by scoring with capsule endoscopy: is transient elastography of clinical impact? J Clin Biochem Nutr 47: 37-44. [Crossref]

7. Mizuno H, Kagaya T, Ooishi N, Takatori H, Yamashita T, et al. (2011) Mucosal findings of the small intestine in patients with portal hypertension due to liver cirrhosis: A capsule endoscopy study. Gastrointest Endosc 53: 1600-1608. [Crossref]

8. Aoyama T, Oka S, Aikata H, Nakano M, Watari I, et al. (2013) Small bowel abnormalities in patients with compensated liver cirrhosis. Dig Dis Sci 58: 1390-1396. [Crossref]

9. De Palma GD, Rega M, Masone S, Persico F, Siciliano S, et al. (2005) Mucosa abnormalities of the small bowel in patients with cirrhosis and portal hypertension: a capsule endoscopy study. Gastrointest Endosc 62: 529-534. [Crossref]

10. Akyuz F, Pinarbasi B, Ermis F, Uyanikoglu A, Demir K, et al. (2010) Is porta hypertensive enteropathy an important additional cause of blood loss in portal hypertensive patients? Scand J Gastroenterol 45: 1497-1502. [Crossref]

11. Matsushita Y, Narahara Y, Fujimori S, Kanazawa H, Itokawa N, et al. (2013) Effects of transjugular intrahepatic portosystemic shunt on changes in the small bowel mucosa of cirrhotic patients with portal hypertension. $J$ Gastroenterol 48: 633-639. [Crossref]
12. Tanihata H, Minamiguchi H, Sato M, Kawai N, Sonomura T, et al. (2009) Changes in portal systemic pressure gradient after balloon-occluded retrograde transvenous obliteration of gastric varices and aggravation of esophageal varices. Cardiovasc Intervent Radiol 32: 1209-1216. [Crossref]

13. Hirota S, Matsumoto S, Tomita M, Sako M, Kono M (1999) Retrograde transvenous obliteration of gastric varices. Radiology 211: 349-356. [Crossref]

14. Koito K, Namieno T, Nagakawa T, Morita K (1996) Balloon-occluded retrograde transvenous obliteration for gastric varices with gastrorenal or gastrocaval collaterals. Am J Roentgenol 167: 1317-1320. [Crossref]

15. Kitamoto M, Imamura M, Kamada K, Aikata H, Kawakami Y, et al. (2002) Balloonoccluded retrograde transvenous obliteration of gastric fundal varices with hemorrhage. AJR Am J Roentgenol 178: 1167-1174. [Crossref]

16. Shibata H, Hashimoto S, Shimizu K, Kawasato R, Shirasawa T, et al. (2015) Beneficia effects of combining computed tomography enteroclysis/enterography with capsule endoscopy for screening tumor lesions in the small intestine. Gastroenterol Res Pract 952787.

17. Kanagawa H, Mima S, Kouyama H, Gotoh K, Uchida T, et al. (1996) Treatment of gastric fundal varices by balloon-occluded retrograde transvenous obliteration. $J$ Gastroenterol Hepatol 11: 51-58. [Crossref]

18. Ishikawa T, Shiratsuki S, Matsuda T, Iwamoto T, Takami T, et al. (2014) Occlusion of portosystemic shunts improves hyperinsulinemia due to insulin resistance in cirrhotic patients with portal hypertension. J Gastroenterol 49: 1333-1341. [Crossref]

19. Perelló A, Escorsell A, Bru C, Gilabert R, Moitinho E, et al. (1999) Wedged hepatic venous pressure adequately reflects portal pressure in hepatitis $C$ virus-related cirrhosis. Hepatology 30: 1393-1397. [Crossref]

20. Akahoshi T, Hashizume M, Tomikawa M, Kawanaka H, Yamaguchi S, et al. (2008) Long-term results of balloon-occluded retrograde transvenous obliteration for gastric variceal bleeding and risky gastric varices: a 10-year experience. $J$ Gastroenterol Hepatol 23: 1702-1709. [Crossref]

21. Takahashi Y, Fujimori S, Narahara Y, Gudis K, Ensaka Y, et al. (2012) Small intestina edema had the strongest correlation with portal venous pressure amongst capsule endoscopy findings. Digestion 86: 48-54. [Crossref]

22. Higashihara H, Urakawa H, Nonokuma SY, Koura SI, Okazaki M, Yoshimitsu K (2010) Influence of balloon-occluded retrograde transvenous obliteration for portosystemic shunts on the liver function. Med Bull Fukuoka Univ 37: 175-180. [Crossref]

23. Fukuda T, Hirota S, Sugimura K (2001) Long-term results of balloon-occluded retrograde transvenous obliteration for the treatment of gastric varices and hepatic encephalopathy. J Vasc Interv Radiol 12: 327-336.

24. Higaki N, Matsui H, Imaoka H, Ikeda Y, Murakami H, et al. (2008) Characteristic endoscopic features of portal hypertensive enteropathy. J Gastroenterol 43: 327-331. [Crossref]

25. Kodama M, Uto H, Numata M, Hori T, Murayama T, et al. (2008) Endoscopic characterization of the small bowel in patients with portal hypertension evaluated by double balloon endoscopy. J Gastroenterol 43: 589-596. [Crossref]

26. Yamamoto H, Sekine Y, Sato Y, Higashizawa T, Miyata T, et al. (2001) Total enteroscopy with a nonsurgical steerable double-balloon method. Gastrointest Endosc 53: 216-220. [Crossref]

Copyright: (C2016 Yokota T. This is an open-access article distributed under the terms of the Creative Commons Attribution License, which permits unrestricted use, distribution, and reproduction in any medium, provided the original author and source are credited. 